\title{
CÁC YẾU TỐ ẢNH HƯỞNG ĐẾN Ý ĐỊ̂H SỬ DỤNG ÚỦ DG DỤNG BAEMIN ĐỂ MUA THỨC ĂN CỦA KHÁCH HÀNG Ở THÀNH PHỐ HỒ CHÍ MINH
}

\author{
NGUYẼ̃N THỊ KIỀU TRANG; NGUYỄN THÀNH LONG; PHẠM NGỌC KIM KHÁNH \\ Trường Đại học Công nghiệp thành phố Hồ Chí Minh, thành phố Hồ Chí Minh \\ phamngockimkhanh@iuh.edu.vn
}

Tóm tắt: Việc đặt đồ ăn qua ứng dụng di động đã không còn xa lạ đối với người dân ở các thành phố lớn, đặc biệt là Thành phố Hồ Chí Minh. Tuy nhiên ứng dụng Baemin không phải là lựa chọn tốt nhất của khách hàng ở Thành phố Hồ Chí Minh với nhiều lý do khác nhau. Do đó, nghiên cứu này đã được tiến hành với mục đích tìm ra các yếu tố ảnh hưởng đến ý định sử dụng ứng dụng Baemin để mua thức ăn của khách hàng. Đối tượng khảo sát là các khách hàng ở Thành phố Hồ Chí Minh có ý định hoặc đang sử dụng ứng dụng Baemin để mua thức ăn. Nghiên cứu định tính được thực hiện thông qua kỹ thuật phỏng vấn chuyên gia trong lĩnh vực có liên quan để hoàn thiện mô hình nghiên cứu đề xuất, bổ sung thang đo và các biến quan sát. Sau đó tiến hành nghiên cứu định lượng với 178 mẫu hợp lệ. Kết quả cho thấy các yếu tố: nhận thức về sự hữu ích, nhận thức dễ sử dụng, ảnh hưởng của xã hội, cảm nhận độ tin cậy, cảm nhận về giá là những yếu tố có tác động đến ý định sử dụng ứng dụng Baemin để mua thức ăn của khách hàng ở Thành phố Hồ Chí Minh. Qua đó đưa ra một số hàm ý quản trị để nâng cao ý định sử dụng ứng dụng Baemin của khách hàng.

Từ khóa: Ý định sử dụng ứng dụng Baemin, ứng dụng Baemin

\section{FACTORS AFFECTING THE INTENTION OF USING BAEMIN APPLICATION TO BUY FOOD OF CUSTOMERS IN HO CHI MINH CITY}

\begin{abstract}
Ordering food via mobile applications is no longer strange to people in big cities, especially Ho Chi Minh City. However, Baemin application is not the best choice of customers in Ho Chi Minh City due to many reasons. Therefore, this study aims to find out the factors affecting the intention to use Baemin application to buy food of customers. The survey subjects are customers in Ho Chi Minh City who intend or are using Baemin app to buy food. Qualitative research is carried out through the technique of interviewing experts in the relevant field to complete the proposed research model, adding scales and observed variables. Then conduct a quantitative study with 178 valid samples. The results show that the factors: perceived usefulness, perceived ease of use, social influence, perceived reliability, perceived price are the factors that affect the intention to use the application. using Baemin to buy food from customers in Ho Chi Minh City. Thereby giving some administrative implications to improve the customer's intention to use Baemin application.
\end{abstract}

Key words: Intention to use Baemin app, Baemin app.

\section{GIỚI THIÊU}

Thức ăn là một trong những nhu cầu cơ bản và thiết yếu nhất của con người. Hiện nay, mọi người ai cũng có những công việc của mình, họ phải làm việc toàn thời gian trong các văn phòng, công trường, xí nghiệp,... do đó việc đi chợ, lựa chọn thực phẩm, mua về và chế biến thành những món ăn là một vấn đề khó khăn và không phải ai cũng có thể sắp xếp ổn thỏa. Những người bận rộn sẵn sàng trả thêm tiền để đặt thức ăn trực tuyến và họ sẽ được giao một cách nhanh chóng thay vì tự làm cơm hộp mang theo hoặc ra ngoài ăn. Cùng với sự phát triển của kinh tế thì tiêu chuẩn của người tiêu dùng ngày càng tăng cao, khắt khe hơn, họ không chỉ muốn ăn ngon mà còn phải có các dịch vụ giao hàng tận nhà. Thấu hiểu tâm lý của khách hàng các công ty đã cho ra đời ứng dụng đặt thức ăn và giao hàng đến tận nhà cho khách hàng. Khách hàng chỉ cần chọn món ăn mà mình thích và đặt hàng sau đó các tài xế sẽ giao hàng nhanh chóng. 
Ngày 16/12/2020, Q\&Me [28] công bố khảo sát về thị trường giao đồ ăn tại Việt Nam. Q\&Me đã tiến hành khảo sát tại Hà Nội và Thành phố Hồ Chí Minh, với sự tham gia của 1046 người từ 18 đến 45 tuổi. Kết quả GrabFood vẫn là ứng dụng được người tiêu dùng sử dụng nhiều nhất, đứng vị trí thứ 2 là Now, kế tiếp là Baemin và GoFood, cuối cùng là Loship. Baemin là ứng dụng xuất hiện muộn nhất so với các ứng dụng khác. Ban đầu Baemin đứng vị trí thứ 4 sau GrabFood, Now và GoFood. Nhưng hiện tại Baemin đã vượt lên vị trí thứ 3 . Baemin có thể ra đời muộn hơn so với các ứng dụng tương tự tại thị trường Việt Nam nhưng Baemin là một đối thủ không thể coi thường khi hiện tại Baemin là ứng dụng giao đồ ăn hàng đầu tại thị trường Hàn Quốc và đang đứng vị trí thứ 3 tại thị trường Việt Nam sau gần 2 năm ra mắt. Liệu Baemin có thể phát triển mạnh mẽ vượt qua GrabFood và Now để vươn lên trở thành ứng dụng giao đồ ăn hàng đầu Việt Nam như đối với thị trường Hàn Quốc? Để đạt được mục tiêu đó Baemin cần nắm bắt được tâm lý của khách hàng, biết được những yếu tố chủ chốt có ảnh hưởng lớn đến ý định sử dụng ứng dụng Baemin của khách hàng để từ đó chú ý phát triển các yếu tố có tác động mạnh mẽ để thu hút sự chú ý của khách hàng thay vì chú ý các yếu tố không quan trọng. Và đó là nguyên nhân tác giả chọn đề tài: "Các yếu tố ảnh hưởng đến ý định sử dụng ứng dụng Baemin để mua thức ăn của khách hàng ở Thành phố Hồ Chí Minh."

\section{CƠ SỞ LÝ THUYÊTT VÀ MÔ HİNH NGHIÊN CÚU}

\subsection{Cơ sở lý thuyết}

\subsubsection{Khái niệm ý định}

Theo Ajzen (1988) [2], cho rằng ý định là một hàm của ba nhân tố: đầu tiên, yếu tố các thái độ đối với hành vi, thứ hai là quy chuẩn chủ quan, thứ ba là nhận thức kiểm soát hành vi. Theo Philips Kotler và cộng sự (2001) [19], cho rằng: trong lúc lựa chọn các phương án mua hàng, khách hàng sẽ đánh giá các phương án lựa chọn và có ý định mua. Đa số quyết định của khách hàng là họ sẽ lựa chọn mặc hàng của thương hiệu mà họ thích nhất. Nhưng sự ảnh hưởng của những người xung quanh và các trường hợp rủi ro có thể cản trở ý định mua trở thành quyết định mua. Theo Elbeck (2008) [9], ý định mua là khả năng mà người tiêu dùng sẵn sàng mua sản phẩm (Elbeck, 2008). Ý định mua của người tiêu dung càng lớn thì khả năng người đó quyết định mua sản phẩm càng cao. Theo Dodds \& cộng sự (1991) [6], ý định mua thể hiện khả năng mà người tiêu dùng mua sản phẩm. Việc khảo sát các yếu tố ảnh hưởng đến ý định mua của khách hàng có ý nghĩa rất quan trọng đối với doanh nghiệp. Từ việc biết được các yếu tố tác động đến ý định mua, doanh nghiệp có thể dự đoán được khả năng mà khách hàng mua sản phẩm.

Ý định sử dụng ứng dụng di động là khả năng người dùng thường xuyên và liên tục sử dụng các ứng dụng trên thiết bị di động trong tương lai (Webster \& cộng sự, 1993; Venkatesh \& Davis, 2000) [26,27].

2.1.2 Úng dụng

Úng dụng là phần mềm được tạo ra để chạy trên các thiết bị thông minh như điện thoại, máy tính bảng và các thiết bị di động khác.

Các ứng dụng thường có sẵn thông qua các nền tảng phân phối ứng dụng, thường được điều hành bởi các chủ sở hữu của hệ điều hành di động như Apple App Store, Google Play, Windows Phone Store, BlackBerry App World. Có những ứng dụng miễn phí những cũng có những ứng dụng bắt buộc phải mua mới có thể tải xuống

\subsection{Lý thuyết liên quan}

\subsubsection{Thuyết hành vi dụ định (Theory Plan of Behavior - TPB)}

Thuyết hành vi dự định (TPB) được phát triển từ thuyết hành động hợp lý (Ajzen \& Fishbein, 1975) [1], Ajzen đã mở rộng thuyết về hành động hợp lý bằng cách thêm nhân tố phi lý trí để tăng tính chính xác cho mô hình dự đoán hành vi. Lý thuyết này được tạo ra để khắc phục sự hạn chế của lý thuyết trước về việc cho rằng hành vi của con người là hoàn toàn do kiểm soát lý trí. Lý thuyết hành vi có kế hoạch hay lý thuyết hành vi dự định là một lý thuyết thể hiện mối quan hệ giữa niềm tin và hành vi của một người nào đó. Trong học thuyết mới này tác giả cho rằng ý định hành vi chịu sự tác động của ba nhân tố: thái độ, chuẩn chủ quan và kiểm soát hành vi.

Thái độ đối với hành vi: là đánh giá của một cá nhân về kết quả sau khi thực hiện một hành vi. Có thể là đánh giá thuận lợi hoặc đánh giá bất lợi về hành vi của cá nhân đó. Chuẩn chủ quan: nhận thức của một cá nhân về áp lực quy phạm xã hội hay nói cách khác là nhận thức của một cá nhân về suy nghĩ của người khác rằng mình nên hay không nên thực hiện một hành vi nào đó, hành vi sẽ bị ảnh hưởng bởi sự phán xét 
của những người quan trọng khác. Kiểm soát hành vi: đây là yếu tố tạo nên sự khác biệt giữa thuyết hành vi dự định và thuyết hành vi hợp lý. Nhận thức về kiểm soát hành vi là nhận thức của một cá nhân về sự dễ dàng hoặc khó khăn trong việc thực hiện hành vi cụ thể.

\subsubsection{Mô hình chấp nhận công nghệ (Technology Acceptance Model - TAM)}

Năm 1985, Davis [4] đã phát triển mô hình chấp nhận công nghệ. Mô hình này cho thấy khả năng chấp nhận của một hệ thống thông tin được xác định bới hai yếu tố chính: nhận thức tính hữu ích và nhận thức dễ sử dụng. Theo như mô hình chấp nhận công nghệ, thói quen sử dụng hệ thống được xác định bởi ý định mà ý định thì được xác định bởi thái độ sử dụng. Sự nhận thức về tính hữu ích và nhận thức dễ sử dụng thì tác động đến thái độ sử dụng. Ngoài ra, Davis còn đưa ra giả thuyết về mối liên hệ giữa nhận thức về tính hữu ích và nhận thức dễ sử dụng.

\subsection{Lược khảo những nghiên cứu trong và ngoài nước có liên quan đến đề tài}

Nguyễn Thị Hồng Hạnh và cộng sự (2020) [14] đã nghiên cứu "Các yếu tố ảnh hưởng đến ý định sử dụng của người tiêu dùng Việt Nam đối với các ứng dụng mua sắm di động." Nghiên cứu tiến hành qua hai giai đoạn: giai đoạn 1 nghiên cứu định tính bằng cách phỏng vấn 10 người có độ tuổi từ 18 - 54 tuồi bao gồm sinh viên, công nhân viên, cán bộ đã sử dụng ứng dụng di động để mua sắm trực tuyến. Giai đoạn 2 nghiên cứu định lượng bằng bảng câu hỏi được gửi qua email và phỏng vấn trực tiếp khách hàng với 315 bảng khảo sát. Kết quả hồi quy cho thấy ý định sử dụng ứng dụng để mua sắm trực tuyến của người tiêu dùng chịu tác động của năm yếu tố: tính linh hoạt, thói quen, tin tưởng, động lực hưởng thụ và rủi ro cảm nhận. Theo San và Dastance (2020) [21], những yếu tố tác động đến ý định đặt hàng giao thức ăn trực tuyến bao gồm chất lượng dịch vụ, nhận thức về sự hữu ích và sự quen thuộc với thương hiệu. Nhận thức về sự hữu ích có tác động cao nhất đến ý định mua hàng, sau đó là sự quen thuộc của thương hiệu và chất lượng dịch vụ. Kết quả nghiên cứu này được sử dụng phương pháp nghiên cứu định lượng, lấy mẫu thuận tiện và thu thập dữ liệu thông qua 304 bảng câu hỏi trực tuyến.

Theo Elango và cộng sự (2018) [8] nghiên cứu "Các yếu tố tác động đến ý định sử dụng ứng dụng giao đồ ăn theo yêu cầu tại Bangkok, Thái Lan.” Nghiên cứu tiến hành khảo sát thông qua 415 bảng câu hỏi trực tuyến tuy nhiên chỉ có 392 bảng câu hỏi phù hợp với nghiên cứu do có câu hỏi sàng lọc nhằm loại bỏ những đối tưởng khảo sát không phù hợp. Kết quả cho thấy yếu tố sự đổi mới của cá nhân và nhận thức về sự hiệu quả có tác động tích cực đến cảm nhận về tính dễ sử dụng, trong khi sự đổi mới của cá nhân và tính dễ sử dụng có tác động tích cực đến nhận thức về sự hữu ích của các ứng dụng giao đồ ăn theo yêu cầu. Yếu tố nhận thức về sự hiệu quả, nhận thức về sự hữu ích và ảnh hưởng của xã hội có tác động tích cực đến ý định sử dụng các ứng dụng giao đồ ăn theo yêu cầu.

\subsection{Giả thuyết nghiên cứu và mô hình nghiên cứu}

\subsubsection{Nhân thức về sư hũu ích}

Nhận thức sự hữu ích là mức độ mà ai đó tin rằng khi họ sử dụng một hệ thống cụ thể sẽ nâng cao hiệu quả thực hiện của họ (Davis, 1985) [4]. Nghiên cứu của Elango và cộng sự (2018) [8] chỉ ra rằng nhận thức về sự hữu ích được xem là một yếu tố có ảnh hưởng đáng kể đến ý định sử dụng các ứng dụng giao đồ ăn theo yêu cầu của người tiêu dùng tại Bangkok, Thái Lan. Nếu người tiêu dùng cảm thấy ứng dụng Baemin giúp họ tối ưu hóa thời gian và họ cảm thấy ứng dụng này rất hữu ích thì họ sẽ hình thành nên ý định sử dụng ứng dụng Baemin. Do đó nhận thức về sự hữu ích của ứng dụng Baemin sẽ có ảnh hưởng trực tiếp đến ý định sử dụng ứng dụng này. Do đó giả thuyết $\mathrm{H} 1$ được đưa ra:

Giả thuyết H1: Nhận thức về sụ hưu ích có ảnh hưởng tích cực (+) đến ý định sủ dụng ứng dụng Baemin để mua thức ăn của khách hàng.

\subsubsection{Nhận thức dễ sư dụng}

Nhận thức dễ sử dụng là khả năng mà cá nhân cho rằng khi họ sử dụng một hệ thống nào đó sẽ không cần cố gắng (Davis, 1985) [4]. Theo như mô hình chấp nhận công nghệ (TAM) của Davis (1993) [5], ta thấy yếu tố nhận thức dễ sử dụng có tác động đến thái độ hành vi và từ thái độ hành vi sẽ có ảnh hưởng đến ý định hành vi của người tiêu dùng. Yếu tố này cũng xuất hiện trong nghiên cứu của Nguyễn Thị Hồng Hạnh và cộng sự (2020) [14]; Rahmaningtyas và cộng sự (2017) [20]; Elango và cộng sự (2018) [8]. Do đó giả thuyết $\mathrm{H} 2$ được đưa ra: 
Giả thuyết H2: Nhận thức dễ sủ dụng có ảnh hưởng tích cưcc (+) đến ý định sủ dụng ứng dụng Baemin để mua thức ăn của khách hàng.

\subsection{3 Ảnh hương của xã hội}

Dựa vào thuyết hành vi dự định của Ajzen (1991) [3], ta thấy chuẩn chủ quan có tác động đến xu hướng hành vi. Chuẩn chủ quan là nhận thức của con người về áp lực xã hội để thể hiện hay không thể hiện hành vi (Ajzen \& Fishbein, 1975) [1]. Ảnh hưởng của xã hội có thể đến từ những người xung quanh. Những người xung quanh sẽ đưa ra lời khuyên nên hay không nên sử dụng, sẽ có tác dụng thúc đẩy hoặc làm giảm ý định của khách hàng. Trong nghiên cứu của Elango và cộng sự (2018) [8], kết quả chỉ ra rằng ảnh hưởng của xã hội có tác động đáng kể đến ý định sử dụng các ứng dụng giao đồ ăn theo yêu cầu của người tiêu dùng tại Bangkok, Thái Lan. Từ đó giả thuyết $\mathrm{H} 3$ được đưa ra:

Giả thuyết H3: Ảnh hưởng của xã hội có ảnh hương tích cục (+) đến ý định sủ dụng ứng dụng Baemin để mua thức ăn của khách hàng.

\subsubsection{Nhận thức về rủi ro}

Người tiêu dùng có xu hướng đánh giá sản phẩm bán thông qua Internet có rủi ro cao. Nguyên nhân là do người mua không biết sản phẩm mình mua thực tế trông như thế nào, rủi ro về tài chính, giao hàng kém chất lượng, rủi ro không giao hàng ảnh hưởng xấu đến thái độ mua sắm trực tuyến (Javadi, 2012) [25]. Người tiêu dùng càng gặp nhiều rủi ro thì càng có thái độ tiêu cực đối với mua sắm trực tuyến dẫn đến ít khả năng mua hàng và ngược lại (Javadi, 2012)[25]. Nhận thức về rủi ro có tác động xấu đến quyết định mua sản phẩm của khách hàng, ta có thể thấy rõ qua nghiên cứu của Nguyễn Thị Hồng Hạnh và cộng sự (2020) [14]. Nghiên cứu của Rahmaningtyas và cộng sự (2017) [20] cũng cho ra kết quả như vậy. Do đó giả thuyết $\mathrm{H} 4$ được hình thành:

Giả thuyết H4: Nhận thức về rủi ro có ảnh hưởng tiêu cực (-) đến ý định sủ̉ dụng ứng dụng Baemin để mua thức ăn của khách hàng.

\subsubsection{Cảm nhận độ tin cậy}

Độ tin cậy được coi là một nhân tố quan trọng góp phần tác động đến quyết định mua hàng thông qua Internet, nghiên cứu của của Nguyễn Thị Hồng Hạnh và cộng sự (2020) [14] đã chứng minh được sự ảnh hưởng của độ tin cậy đối với ý định mua sắm thông qua Internet của người tiêu dùng. Thông tin chi tiết về giá sản phẩm và phí giao hàng sẽ là một trong những yếu tố giúp người tiêu dùng tin tưởng và an tâm giao dịch hơn, nó cũng giúp người tiêu dùng cảm nhận được sự tin cậy khi mua hàng trực tuyến. Do đó giả thuyết H5 được đưa ra:

Giả thuyết H5: Cảm nhận độ tin cậy có ảnh huơong tích cực (+) đến ý định sủ dụng ưng dụng Baemin để mua thức ăn của khách hàng.

\subsubsection{Cảm nhận về giá}

Giá cả được xem là yếu tố quan trọng có ảnh hưởng đến ý định sử dụng ứng dụng Baemin của khách hàng. Như trong nghiên cứu của Rahmaningtyas và cộng sự (2017) [20] đã chứng minh được điều đó. Internet phát triển đã giúp cho khách hàng dễ dàng biết được giá cả của những mặc hàng mà họ định mua, họ sẽ đánh giá chi phí vận chuyển giữa các thương hiệu và người tiêu dùng sẽ chọn giá mà họ cảm thấy rẻ hơn với chất lượng tốt hơn. Vì vậy giả thuyết $\mathrm{H} 6$ được đưa ra:

Giả thuyết H6: Cảm nhận về giá có ảnh hưởng tích cực (+) đến ý định sủ dụng ưng dụng Baemin để mua thức ăn của khách hàng. 


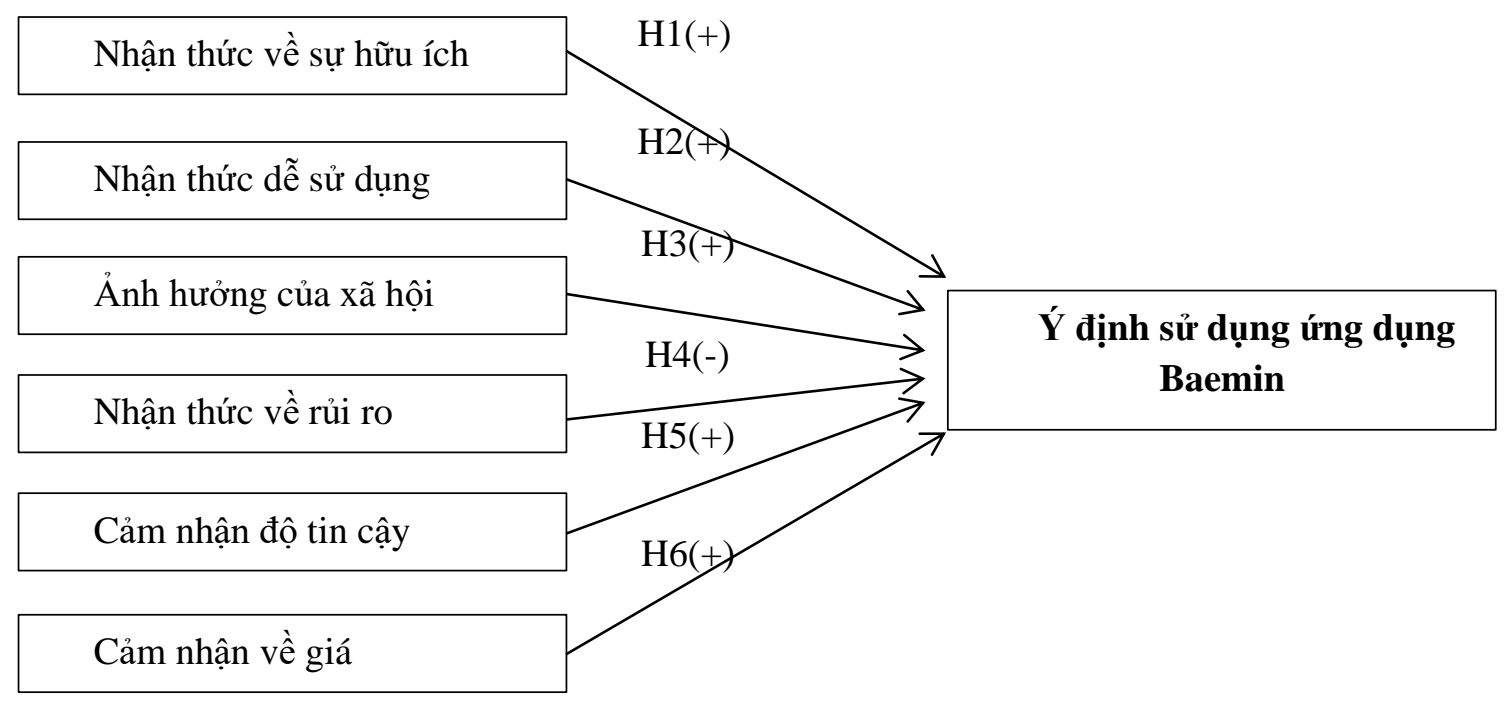

Hình 1: Mô hình nghiên cứu đề xuất

Nguồn: Tác giả tổng hợp tù̀ các nghiên cưu trước

\section{PHƯƠNG PHÁP NGHIÊN CÚU}

Tác giả đã tiến hành khảo sát 250 khách hàng ở Thành phố Hồ Chí Minh với mục đích thu thập số liệu. Sau khi thu thập đủ, tác giả sẽ mã hóa và loại bỏ các dữ liệu không đủ tiêu chuẩn, tiếp theo tác giả xử lý dữ liệu bằng SPSS bao gồm các bước: thống kê mô tả, chạy Cronbach's Alpha, chạy EFA để kiểm định giá trị hội tụ và giá trị phân biệt của các biến thành phần, sau đó tiến hành chạy tương quan, hồi quy.

\subsection{Phương pháp chọn mẫu}

Mẫu là những người có ý định sử dụng ứng dụng Baemin tại Thành phố Hồ Chí Minh với giới tính, nghề nghiệp, thu nhập,...khác nhau. Theo Hair và cộng sự (2010) [10], trong phân tích nhân tố thì số quan sát ít nhất là 5 lần số biến quan sát. Trong mô hình nghiên cứu có 30 biến quan sát, vì vậy tối thiểu cần $\mathrm{n}=5 \mathrm{x} 30$ $=150$ mẫu. Tác giả chọn số lượng mẫu trong nghiên cứu chính thức là 250 được xem là đủ lớn để thỏa mãn điều kiện và phòng trường hợp các mẫu không đủ tiêu chuẩn và bị loại bỏ.

\subsection{Thang đo nghiên cứu}

\begin{tabular}{|c|c|c|}
\hline Nhân tố & Biến quan sát & Nguồn \\
\hline \multirow{5}{*}{$\begin{array}{l}\text { Nhận } \\
\text { thức về } \\
\text { sự hữu } \\
\text { ích }\end{array}$} & $\begin{array}{l}\text { Sử dụng ứng dụng di động mua thức ăn giúp tôi tiết kiệm thời gian và } \\
\text { công sức hơn }\end{array}$ & $\begin{array}{l}\text { Tác giả đề xuất từ ý kiến } \\
\text { chuyên gia }\end{array}$ \\
\hline & So sánh được nhiều sản phẩm & \multirow{3}{*}{$\begin{array}{l}\text { Rahmaningtyas và cộng sự } \\
\text { (2017) [20] }\end{array}$} \\
\hline & Nhận được nhiều thông tin & \\
\hline & Nhận thông tin nhanh hơn & \\
\hline & $\begin{array}{l}\text { Tôi thấy thật hữu ích khi sử dụng ứng dụng di động trong việc mua } \\
\text { thức ăn }\end{array}$ & $\begin{array}{l}\text { Tác giả đề xuất từ ý kiến } \\
\text { chuyên gia }\end{array}$ \\
\hline \multirow{6}{*}{$\begin{array}{l}\text { Nhận } \\
\text { thức dễ } \\
\text { sử dụng }\end{array}$} & Mua mà không cần trợ giúp nhiều & \multirow{4}{*}{$\begin{array}{l}\text { Rahmaningtyas và cộng sự } \\
\text { (2017) [20] }\end{array}$} \\
\hline & Ít nỗ lực thể chất hơn & \\
\hline & Hệ thống thanh toán dễ dàng & \\
\hline & Tìm sản phẩm dễ dàng & \\
\hline & Học cách mua sắm trên ứng dụng di động thật dễ dàng đối với tôi & \multirow[b]{2}{*}{$\begin{array}{l}\text { Nguyễn Thị Hồng Hạnh và } \\
\text { cộng sự (2020) [14] }\end{array}$} \\
\hline & $\begin{array}{l}\text { Thật dễ dàng theo dõi thông báo về tin khuyến mãi, thông tin đơn } \\
\text { hàng qua ứng dụng di động. }\end{array}$ & \\
\hline
\end{tabular}



THỬC ĂN CỦA KHÁCH HÀNG Ở THÀNH PHỐ HỒ CHÍ MINH

\begin{tabular}{|c|c|c|}
\hline \multirow{3}{*}{$\begin{array}{c}\text { Ảnh } \\
\text { hưởng } \\
\text { của xã } \\
\text { hội }\end{array}$} & $\begin{array}{l}\text { Người dùng nghĩ rằng mua hàng trực } \\
\text { tuyến phù hợp với xu hướng hiện tại }\end{array}$ & $\begin{array}{l}\text { Đoàn Thị Thu Trang } \\
\text { (2020) [7] }\end{array}$ \\
\hline & $\begin{array}{l}\text { Những người có ảnh hưởng đến tôi, } \\
\text { nghĩ rắng tôi nên mua hàng trực tuyến } \\
\text { trong các cửa hàng }\end{array}$ & $\begin{array}{l}\text { NathaliePeña-García } \\
\text { và cộng sự (2020) } \\
\text { [17] }\end{array}$ \\
\hline & $\begin{array}{l}\text { Người thân, bạn bè, đồng nghiệp của tôi } \\
\text { đang sử dụng ứng dụng Baemin để mua } \\
\text { hàng }\end{array}$ & $\begin{array}{l}\text { Tác giả đề xuất từ ý } \\
\text { kiến chuyên gia }\end{array}$ \\
\hline \multirow{4}{*}{$\begin{array}{l}\text { Nhận } \\
\text { thức về } \\
\text { rủi ro }\end{array}$} & Thanh toán trực tuyến đáng tin cậy & \multirow{4}{*}{$\begin{array}{l}\text { Rahmaningtyas và } \\
\text { cộng sự (2017) [20] }\end{array}$} \\
\hline & Sản phẩm như mong đợi & \\
\hline & Đóng gói đặc biệt & \\
\hline & Giao hàng đúng hạn & \\
\hline \multirow{4}{*}{$\begin{array}{c}\text { Cảm } \\
\text { nhận } \\
\text { độ tin } \\
\text { cậy }\end{array}$} & Thông tin sản phẩm đáng tin cậy & $\begin{array}{l}\text { Rahmaningtyas và } \\
\text { cộng sự (2017) [20] }\end{array}$ \\
\hline & $\begin{array}{l}\text { Thông tin nhân viên giao hàng đáng tin } \\
\text { cậy }\end{array}$ & $\begin{array}{l}\text { Tác giả đề xuất từ ý } \\
\text { kiến chuyên gia }\end{array}$ \\
\hline & $\begin{array}{l}\text { Tôi nghĩ rằng thông tin cá nhân sẽ được } \\
\text { bảo mật khi sử dụng ứng dụng di động }\end{array}$ & \multirow{2}{*}{$\begin{array}{l}\text { Nguyễn Thị Hồng } \\
\text { Hạnh và cộng sự } \\
(2020)[14]\end{array}$} \\
\hline & $\begin{array}{l}\text { Tôi cảm thấy tin tưởng ứng dụng di } \\
\text { động khi mua sắm }\end{array}$ & \\
\hline \multirow{4}{*}{$\begin{array}{l}\text { Cảm } \\
\text { nhận } \\
\text { về giá }\end{array}$} & Giá cả phải chăng & \multirow{2}{*}{$\begin{array}{l}\text { Rahmaningtyas và } \\
\text { cộng sự (2017) [20] }\end{array}$} \\
\hline & Giá theo chất lượng & \\
\hline & Chương trình giảm giá hấp dẫn & \multirow{2}{*}{$\begin{array}{l}\text { Tác giả đề xuất từ ý } \\
\text { kiến chuyên gia }\end{array}$} \\
\hline & Giá được trình bày rõ ràng & \\
\hline \multirow{4}{*}{ Ý định } & $\begin{array}{l}\text { Tôi đang suy nghĩ về việc sử dụng ứng } \\
\text { dụng Baemin đề mua thức ăn }\end{array}$ & \multirow{3}{*}{$\begin{array}{l}\text { Tác giả đề xuất từ ý } \\
\text { kiến chuyên gia }\end{array}$} \\
\hline & $\begin{array}{l}\text { Tôi có ý định sẽ sử dụng ứng dụng } \\
\text { Baemin để mua thức ăn }\end{array}$ & \\
\hline & $\begin{array}{l}\text { Tôi chắc chắn sẽ sử dụng ứng dụng } \\
\text { Baemin để mua thức ăn. }\end{array}$ & \\
\hline & $\begin{array}{l}\text { Tôi sẽ giới thiệu người khác mua hàng } \\
\text { trên ứng dụng di động mà tôi dùng }\end{array}$ & $\begin{array}{l}\text { Nguyễn Thị Hồng } \\
\text { Hạnh và cộng sự } \\
\text { (2020) [14] }\end{array}$ \\
\hline
\end{tabular}

\subsection{Thu thập dữ liệu}

Nguồn: Tác giả dụa vào nghiên cứu trước và đề xuất theo ý kiến chuyên gia

Sau khi khảo sát tác giả thu được 250 câu trả lời, trong đó có 178 câu trả lời hợp lệ dùng làm dữ liệu nghiên cứu. Tác giả đã thống kê mô tả mẫu như sau:

Bảng 1: Thống kê mô tả mẫu theo đặc điểm cá nhân

\begin{tabular}{|c|l|c|c|}
\hline Đặc điểm cá nhân & \multicolumn{1}{|c|}{ Chi tiết } & Số lượng & Tỷ lệ (\%) \\
\hline \multirow{3}{*}{ Giới tính } & Nam & 78 & 43,8 \\
\cline { 2 - 4 } & Nữ & 100 & 56,2 \\
\hline \multirow{3}{*}{ Độ tuổi } & Từ $18-25$ & 116 & 65,2 \\
\cline { 2 - 4 } & Từ $26-35$ & 52 & 29,2 \\
\cline { 2 - 4 } & Từ $36-50$ & 10 & 5,6 \\
\cline { 2 - 4 } & Trên 50 & 0 & 0 \\
\hline Nghề nghiệp & Nội trợ & 2 & 1,1 \\
\hline
\end{tabular}



THỨC ĂN CỦA KHÁCH HÀNG Ở THÀNH PHỐ HỒ CHÍ MINH

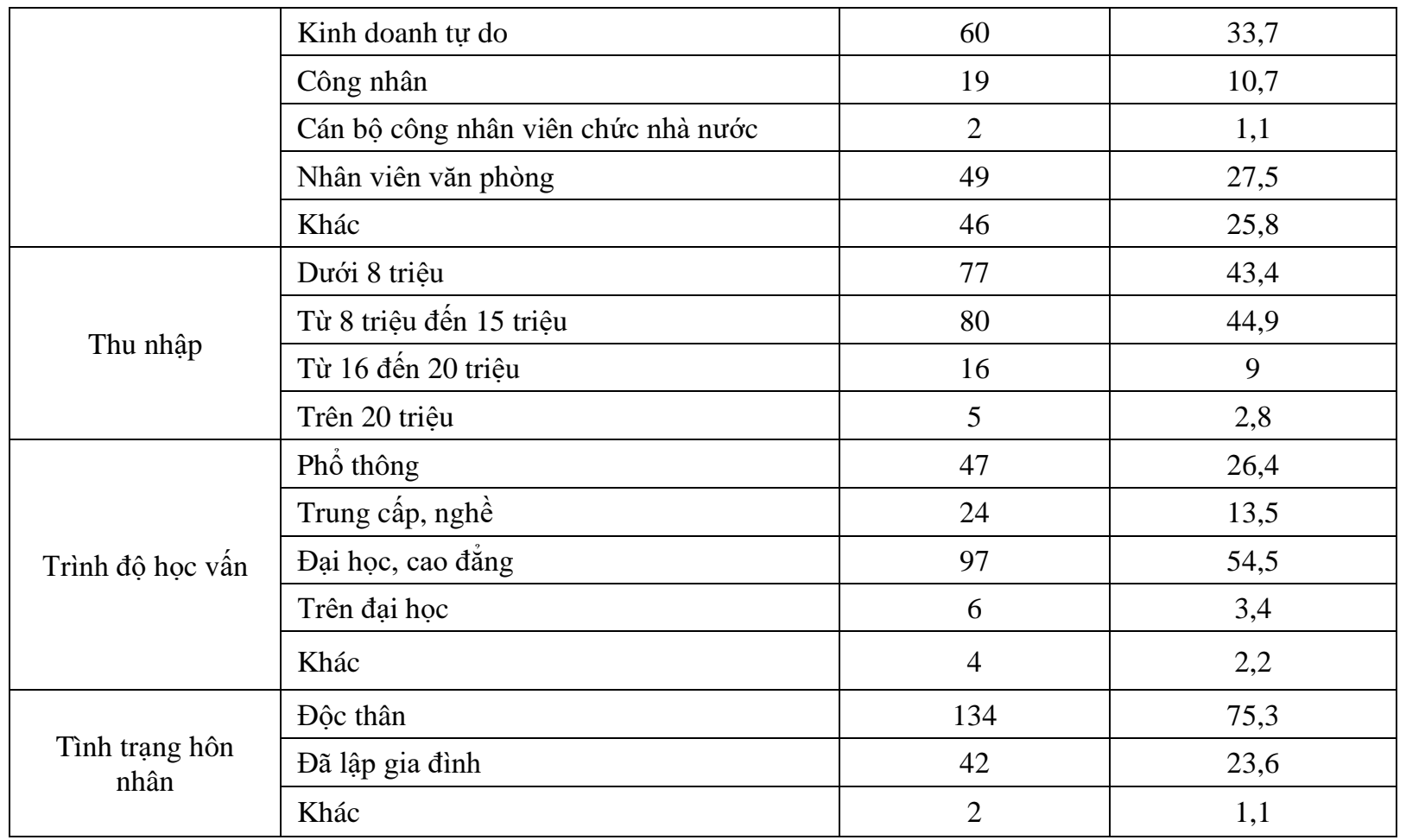

Nguồn: Tác giả tổng hợp tù kết quả phân tích dũ liệu

Theo bảng 1 thì ta thấy được, giới tính nữ cao hơn so với giới tính nam, độ tuổi chiếm tỷ lệ cao nhất là từ $18-25$, kinh doanh tự do là nghề nghiệp được lựa chọn nhiều nhất, đa số có thu nhập từ 8 triệu đến 15 triệu, trình độ học vấn nhiều nhất là đại học, cao đẳng, độc thân chiếm tỷ lệ cao nhất trong tình trạng hôn nhân.

\section{KẾT QUẢ NGHIÊN CÚU VÀ THẢO LUẬN}

\subsection{Kết quả phân tích độ tin cậy của thang đo}

Bảng 2 cho ta thấy, tất cả các yếu tố đều có hệ số tin cậy Cronbach Alpha lớn hơn 0,6. Hệ số tương quan biến tổng của các biến quan sát đều lớn hơn 0,3 . Vì vậy, tất cả 26 biến quan sát thuộc thang đo các thành phần và 4 biến quan sát thuộc thang đo ý định đều đạt độ tin cậy.

Bảng 2: Kết quả phân tích Cronbach's Alpha

\begin{tabular}{|c|c|c|c|}
\hline Thang đo thành phần & $\begin{array}{c}\text { Số biến quan sát } \\
\text { ban đầu }\end{array}$ & Hệ số Cronbach's Alpha & $\begin{array}{c}\text { Hệ số tương quan biến } \\
\text { tồng }\end{array}$ \\
\hline Nhận thức về sự hữu ích & 5 & 0,904 & $\geq 0,662$ \\
\hline Nhận thức dễ sử dụng & 6 & 0,914 & $\geq 0,735$ \\
\hline Ảnh hưởng của xã hội & 3 & 0,746 & $\geq 0,493$ \\
\hline Nhận thức về rủi ro & 4 & 0,891 & $\geq 0,728$ \\
\hline Cảm nhận độ tin cậy & 4 & 0,870 & $\geq 0,520$ \\
\hline Cảm nhận về giá & 4 & 0,757 & $\geq 0,506$ \\
\hline Ý định & 4 & 0,760 & \\
\hline
\end{tabular}

\subsection{Kết quả phân tích nhân tố khám phá (EFA)}

Nguồn: Tác giả tổng hợp tù kết quả phân tích dũ liệu

Bảng 3: Kết quả phân tích EFA

\begin{tabular}{|c|c|c|c|c|}
\hline Nhân tố & $\begin{array}{c}\text { Hệ số } \\
\text { KMO }\end{array}$ & Sig & Tổng phương sai trích & $\begin{array}{c}\text { Hệ số Factor } \\
\text { Loading }\end{array}$ \\
\hline 1. Các biến độc lập & 0,808 & 0,000 & 70,666 & \\
\hline
\end{tabular}



THỨC ĂN CỦA KHÁCH HÀNG Ở THÀNH PHỐ HỒ CHÍ MINH

\begin{tabular}{|l|l|l|l|l|}
\hline Nhận thức về sự hữu ích & & & & $0,756-0,881$ \\
\hline Nhận thức dễ sử dụng & & & & $0,803-0,855$ \\
\hline Ảnh hưởng của xã hội & & & & $0,744-0,857$ \\
\hline Nhận thức về rủi ro & & & & $0,840-0,874$ \\
\hline Cảm nhận độ tin cậy & & & & $0,826-0,877$ \\
\hline Cảm nhận về giá & & & & $0,736-0,768$ \\
\hline 2. Biến phụ thuộc & 0,732 & 0,000 & 58,461 & \\
\hline \multicolumn{1}{|c|}{ Ý định } & & & & $0,716-0,846$ \\
\hline
\end{tabular}

Nguồn: Tác giả tổng hợp tùu kết quả phân tích dũ liệu

Qua bảng 3, ta thấy biến độc lập có hệ số $\mathrm{KMO}=0,808>0,5$, chứng tỏ kết quả phân tích yếu tố đạt độ tin cậy. Ta cũng thấy hệ số Sig có giá trị là $0,000<0,05$ thể hiện rằng kết quả phân tích yếu tố đảm bảo được mức ý nghĩa thống kê. Giá trị Eigenvalues (Đại diện cho sự biến thiên được giải thích bởi mẫu số) =1,893> 1 biến quan sát được nhóm lại thành 6 nhân tố. Tổng phương sai trích là 70,666\%, tức là giải thích được $70,666 \%$ sự biến thiên của dữ liệu.

Biến phụ thuộc: Hệ số KMO $=0,732>0,5$ cho thấy kết quả phân tích yếu tố đạt độ tin cậy. Kiểm định Bartlett's Test có hệ số sig $=0,000<0,05$ thể hiện rằng kết quả phân tích yếu tố đảm bảo được mức ý nghĩa thống kê. Phân tích phương sai bằng $58,461 \%$ giải thích được $58,461 \%$ sự biến thiên của dữ liệu và giá trị Eigenvalues là 2,338> 1 .

\subsection{Kết quả phân tích hồi quy và kiểm định giả thuyết}

$\mathrm{R}^{2}$ có giá trị Sig rất nhỏ $(\mathrm{Sig}=0,000)$ cho thấy mô hình hồi quy phù hợp. Cả 5 yếu tố giải thích $72 \%$ sự biến thiên của dữ liệu. Các hệ số Tolerance là khá cao đều từ $0,946-0,976$ và các hệ số VIF đều dưới 10 cho thấy không có sự đa cộng tuyến giữa các biến độc lập.

Kết quả ở bảng 5 cho thấy tất cả các biến độc lập đều tác động cùng chiều đến ý định sử dụng Baemin có ý nghĩa thống kê với độ tin cậy $95 \%$ theo mức độ giảm dần là: Nhận thức về sự hữu ích $(\beta=0,436)$; Ảnh hưởng của xã hội $(\beta=0,363)$; Nhận thức dễ sử dụng $(\beta=0,343)$; Cảm nhận độ tin cậy $(\beta=0,247)$; Cảm nhận về giá $(\beta=0,234)$.

Bảng 4: Kết quả phân tích hồi quy

\begin{tabular}{|c|c|c|c|c|c|c|c|c|}
\hline & \multirow{2}{*}{ Mô hình } & \multicolumn{2}{|c|}{$\begin{array}{c}\text { Hệ số hồi quy chưa } \\
\text { chuẩn hóa }\end{array}$} & \multirow{2}{*}{$\begin{array}{c}\text { Hệ số hồi quy } \\
\text { chuẩn hóa }\end{array}$} & \multirow{2}{*}{$\begin{array}{c}\text { Giá trị } \\
\text { kiểm định } \\
\mathbf{t}\end{array}$} & \multirow{2}{*}{$\begin{array}{c}\text { Mức ý } \\
\text { nghĩa Sig }\end{array}$} & \multicolumn{2}{|c|}{ Thống kê đa cộng tuyến } \\
\hline & & $\mathbf{B}$ & $\begin{array}{l}\text { Sai số } \\
\text { chuẩn }\end{array}$ & & & & Chấp nhận & VIF \\
\hline \multirow{6}{*}{1} & Hằng số & $-0,778$ & 0,236 & & $-3,290$ & 0,001 & & \\
\hline & NTVSHI & $\mathbf{0 , 3 3 3}$ & 0,032 & 0,436 & 10,522 & 0,000 & 0,947 & 1,056 \\
\hline & NTDSD & 0,278 & 0,034 & 0,343 & 8,282 & $\mathbf{0 , 0 0 0}$ & 0,949 & 1,054 \\
\hline & AHCXH & 0,253 & 0,028 & 0,363 & 8,893 & $\mathbf{0 , 0 0 0}$ & 0,976 & 1,024 \\
\hline & CNĐTC & $\mathbf{0 , 1 7 1}$ & 0,028 & 0,247 & 6,026 & 0,000 & 0,964 & 1,037 \\
\hline & CNVG & 0,219 & 0,039 & 0,234 & 5,654 & 0,000 & 0,946 & 1,057 \\
\hline
\end{tabular}

Nguồn: Kết quả phân tích dũ liệu của tác giả

Ta có phương trình hồi quy chuẩn hóa mô tả sự biến động của các nhân tố ảnh hưởng đến ý định sử dụng ứng dụng Baemin để mua thức ăn của khách hàng ở TP.HCM:

$\mathrm{YD}=0,436 * \mathrm{NTVSHI}+0,343 * \mathrm{NTDSD}+0,363 * \mathrm{AHCXH}+0,247 * \mathrm{CN} \mathrm{NTC}+0,234 * \mathrm{CNVG}$

Từ phương trình trên ta thấy nhân tố nhận thức về sự hữu ích tác động mạnh nhất tới ý định sử dụng ứng dụng Baemin, tiếp theo là yếu tố ảnh hưởng của xã hội, thứ ba là nhân tố nhận thức dễ sử dụng, thứ tư là nhân tố cảm nhận độ tin cậy và cuối cùng là nhân tố cảm nhận về giá. 


\section{HÀM Ý QUẢN TRI}

Thư nhất, yếu tố nhận thức về sự hữu ích có tác động mạnh nhất đến ý định sử dụng ứng dụng Baemin để mua thức ăn của khách hàng ở Thành phố Hồ Chí Minh với $\beta=0,436$. Theo kết quả thống kê các biến quan sát có giá trị lần lượt là: $\operatorname{NTVSHI} 1(3,79), \operatorname{NTVSHI} 2(3,80), \operatorname{NTVSHI} 3(3,87), \operatorname{NTVSHI} 4(3,82)$, NTVSHI5 $(3,74)$. Điều đó cho thấy khách hàng nhận thấy ứng dụng Baemin hữu ích. Một số hàm ý được đưa ra như sau: Có kế hoạch quảng cáo đánh vào sự tiện lợi của ứng dụng Baemin, ưu điểm nổi bật của ứng dụng Baemin so với các ứng dụng tương tự khác. Tạo mục so sánh giá, đánh giá dịch vụ và chi phí giao hàng giữa 2 cửa hàng để giúp người mua dễ dàng so sánh giá cả, chât lượng dịch vụ, chất lượng của món ăn.

Thư hai, yếu tố ảnh hưởng của xã hội có tác động đến ý định sử dụng ứng dụng Baemin của khách hàng với $\beta=0,363$. Theo kết quả thống kê các biến quan sát có giá trị lần lượt là: $\operatorname{AHCXH1}(3,78)$, $\mathrm{AHCXH} 2(3,49), \mathrm{AHCXH3}(3,62)$. Qua đó ta thấy yếu tố xã hội có ảnh hưởng đến ý định sử dụng ứng dụng Baemin, đặc biệt là những người xung quanh. Một số hàm ý được đưa ra như sau: Nên đưa ra các chương trình ưu đãi khi khách hàng giới thiệu ứng dụng Baemin cho những người xung quanh sử dụng. Có chiến lược marketing rộng rãi để ứng dụng Baemin trở thành một thương hiệu quen thuộc đối với khách hàng. Đồng thời tạo hình ảnh thương hiệu đẹp trong lòng khách hàng để khách hàng không chỉ sử dụng ứng dụng Baemin để mua thức ăn mà còn giới thiệu cho những người xung quanh cùng sử dụng.

Thứ $b a$, yếu tố nhận thức dễ sử dụng có tác động tới ý định sử dụng ứng dụng Baemin của khách hàng với $\beta=0,343$. Theo kết quả thống kê các biến quan sát có giá trị lần lượt là: $\operatorname{NTDSD1}(3,89), \operatorname{NTDSD} 2(3,88)$, NTDSD3(3,87), NTDSD4(3,84), NTDSD5 $(3,88)$, NTDSD6 $(3,90)$. Chứng tỏ người sử dụng có quan tâm ứng dụng Baemin có dễ sử dụng không. Vì vậy giao diện của ứng dụng Baemin nên được xây dựng đơn giản, dễ nhìn. Các chương trình giảm giá được liệt kê cụ thể, ghi rõ các điều kiện áp dụng để tránh sự nhầm lẫn cho người sử dụng. Có hướng dẫn sử dụng ứng dụng Baemin cho khách hàng, đặc biệt là những người ít tiếp xúc với các công nghệ mới để họ có thể dễ dàng sử dụng các chức năng trên ứng dụng để mua thức ăn. Khi có khuyến mãi thì ứng dụng Baemin nên tạo thông báo để cho khách hàng biết chứ không cần khách hàng phải vào ứng dụng mới biết được có chương trình khuyến mãi.

Thư tux, yếu tố cảm nhận độ tin cậy cũng là một yếu tố có tác động tích cực đến ý định sử dụng ứng dụng Baemin của khách hàng với $\beta=0,247$. Theo kêt quả thống kê các biến quan sát có giá trị lần lượt là: CNĐTC1 $(3,76), \operatorname{CNĐTC2}(3,88), \operatorname{CNĐTC} 3(3,85), \mathrm{CN} Đ T C 4(3,85)$. Qua đó ta thấy người sử dụng có quan tâm đến độ tin cậy của ứng dụng Baemin. Do đó nhà quản trị cần: cam kết bảo mật thông tin của khách hàng, đảm bảo không bán hoặc tiết lộ thông tin của khách hàng dưới mọi hình thức. Bên cạnh đó ứng dụng Baemin nên cập nhật hình ảnh thực tế của các món ăn và cập nhật đầy đủ hình ảnh để tránh việc chỉ có tên món ăn mà không có hình ảnh hoặc hình ảnh khác xa với thực tế của món ăn. Khi khách hàng nhận món sẽ thấy thất vọng vì món ăn không giống với hình ảnh trên ứng dụng, khi đã mất lòng tin thì khách hàng sẽ không sử dụng ứng dụng Baemin nữa. Thông tin của nhân viên giao thức ăn cũng phải được trình bày rõ ràng, minh bạch.

Thư năm, yếu tố cảm nhận về giá cũng là một yếu tố có tác động tích cực đến ý định sử dụng ứng dụng Baemin của khách hàng với $\beta=0,234$. Theo kết quả thống kê các biến quan sát có giá trị lần lượt là: CNVG1(3,78), CNVG2(3,87), CNVG3(4,06), CNVG4(4,03). Điều đó cho thấy khách hàng có chú ý đến giá khi có ý định sử dụng ứng dụng Baemin. Một số hàm ý quản trị được đưa ra như sau: Ứng dụng Baemin nên được thiết lập các công cụ sắp xếp theo giá để người mua có thể xem giá các món ăn từ thấp đến cao hoặc ngược lại. Doanh nghiệp nên tạo thêm mục khách hàng thân thiết trong ứng dụng Baemin để khi khách hàng đặt món qua ứng dụng này sẽ được tích điểm và sẽ được giảm giá trong lần mua tiếp theo. Việc này không những có thể giúp ứng dụng Baemin được nhiều người sử dụng hơn mà nó còn biến họ thành những vị khách trung thành của doanh nghiệp.

\section{HẠN CHẾ CỦA NGHIÊN CÚ̉U VÀ ĐỀ XUÂT HƯỚNG NGHIÊN CÚU TIẾP THEO}

\subsection{Hạn chế của nghiên cứu}

Bên cạnh việc đạt được các mục tiêu đề ra, nghiên cứu vẫn còn một số mặt hạn chế: đầu tiên, tác giả đã sử dụng phương pháp lấy mẫu ngẫu nhiên thuận tiện nên dữ liệu thu thập không hoàn toàn đại diện cho tổng thể. Ngoài ra, số lượng mẫu hợp lệ của bài nghiên cứu là 178 mẫu, là quá nhỏ so với tổng số dân của Thành phố Hồ Chí Minh. Hạn chế thứ hai là nghiên cứu được thực hiện với các đối tượng khảo sát trong phạm vi 
Thành phố Hồ Chí Minh do đó mức độ tổng quát của kết quả bị giới hạn và không phản ánh chính xác được. Hạn chế cuối cùng là tác giả mới chỉ tìm ra được 5 yếu tố ảnh hưởng đến ý định sử dụng ứng dụng Baemin để mua thức ăn. Nhưng trên thực tế còn rất nhiều những yếu tố khác cũng ảnh hưởng đến ý định sử dụng ứng dụng Baemin để mua thức ăn của khách hàng.

\section{2 Đề xuất hướng nghiên cứu tiếp theo}

Từ những hạn chế đã được nêu trên, tác giả đã đưa ra một số đề xuất cho các nghiên cứu sau như:

Thư nhất, nên mở rộng phạm vi nghiên cứu rộng ra và nâng kích thước mẫu khảo sát lớn hơn để kết quả nghiên cứu mang tính đại diện và khái quát hơn.

Thư hai, các nghiên cứu sau có thể sử dụng phương pháp lấy mẫu theo xác suất và kết hợp với phương pháp lấy mẫu theo tỷ lệ để tăng tính khái quát cho bài nghiên cứu.

Thứ $b a$, thực hiện bước nghiên cứu định tính kĩ lưỡng hơn để có thể đề xuất thêm nhiều yếu tố ảnh hưởng đến ý định sử dụng ứng dụng Baemin để mua thức ăn của khách hàng.

\section{TÀI LIỆU THAM KHẢO}

[1]. Ajzen, I., \& Fishbein, M. (1975). Belief, Attitude, Intention, and Behavior: An Introduction to Theory and Research. USA: Addison-Wesley Publishing Company.

[2]. Ajzen, I. (1988).Attitudes, Personality, and Behavior. Chicago: Dorsey press.

[3]. Ajzen, I. (1991). The Theory of Planned Behavior. Organizational behavior and human decision processes, 50(2), 179-211

[4]. Davis, F. D. (1985). A Technology Acceptance Model for Empirically Testing New End-User Information Systems: Theory and Results. Massachusetts Institute of Technology.

[5]. Davis, F. D. (1993). User acceptance of information technology: system characteristics, user perceptions and behavioral impacts, International journal of man-machine studies, 38(3), 475-487.

[6]. Dodds, W.B., Monroe, K.B. and Grewal, D. (1991). Effects of Price, Brand, and Store Information on Buyers' Product Evaluations. Journal of Marketing Research, 28, 307-319.

[7]. Đoàn Thị Thu Trang (2020). Factors affecting online purchase intention: A study of Vietnam online customers. Management Science Letters, 10(10), 2337-2342.

[8]. Elango, D., Dowpiset, K., \& Chantawaranurak, J. (2018). A Study on Factors Impacting Consumers' Intention to Use On-demand Food Delivery Applications in Bangkok, Thailand. Truy xuất từ: https://ssrn.com/abstract=3321587

[9]. Elbeck, M. \& Tirtiroglu, E. (2008). Qualifying Purchase Intention Using Queueing Theory, Journal of applied quantitative methods, 3(2),167-169.

[10]. Hair, J., Black, W., Babin, B., \& Anderson, R. (1998). Multivariate Data Analysis with Readings. Englewood Cliffs, NJ: Prentice-Hall.

[11]. Khánh Khiêm, (2021, ngày 29 tháng 1). Baemin là gì? Uu và nhược điểm của ứng dụng giao đồ ăn số 1 Hàn Quốc. Truy xuất từ: https://marketingai.admicro.vn/baemin-la-gi-uu-va-nhuoc-diem-cua-ung-dung-giao-doan-so-1-han-quoc/

[12]. Nguyễn Đình Thọ \& Nguyễn Thị Mai Trang (2007). Nghiên cứu Khoa học Marketing ứng dụng mô hình cấu trúc tuyến tính SEM. TP.HCM: Nhà xuất bản ĐH Quốc gia.

[13]. Nguyễn Minh Tuấn, Hà Trọng Quang, \& Nguyễn Vũ Vân Anh. (2015). Xử lý dũ liệu nghiên cúu SPSS for windows. TP.HCM: Nhà xuất bản ĐH Công nghiệp Thành phố Hồ Chí Minh.

[14]. Nguyễn Thị Hồng Hạnh, Dương Minh Hùng, Vũ Duy Phương Trinh, Cao Trương Tường Vy, \& La Thị Như Ý (2020). Các yếu tố ảnh hưởng đến ý định sử dụng ứng dụng di động để mua sắm trực tuyến tại Thành phố Hồ Chí Minh. Tạp chí Phát triển Khoa học và Công nghệ - Kinh tế-Luật và Quản lý, 3(4), 390-401.

[15]. Nhã Vy, (2020, ngày 21 tháng 12). Baemin bưt phá trên thị trương giao đồ ăn, GrabFood vấn đúng đầu. Truy xuất từ: https://taichinhdoanhnghiep.net.vn/baemin-but-pha-tren-thi-truong-giao-do-an-grabfood-van-dungdau-d17641.html

[16]. Nunnally, J. C., \& Bernstein, I. H. (1994). The Assessment of Reliability. Psychometric Theory, 3, $248-292$.

[17]. Peña-García, N., Gil-Saura, I., Rodríguez-Orejuela, A., \& Siqueira-Junior, J. R. (2020). Purchase intention and purchase behavior online: A cross-cultural approach. Heliyon. 6(6).

[18]. doi: 10.1016/j.heliyon.2020.e04284.

[19]. Philip Kotler. (2001). Quản trị Marketing. Nhà xuất bản Thống Kê.

[20]. Rahmaningtyas, A., Hartono, S., \& Suryantini, A. (2017). Factors affecting online purchasing of local food. Agro Ekonomi, 28(2), 189-204. 
[21]. San, S. S., \& Dastane, O. (2021). Key Factors Affecting Intention to Order Online Food Delivery. Journal of Industrial Distribution \& Business, 12(2), 19-27.

[22]. Tabachnick, B. G., \& Fidell, L. S. (1996). Using Multivariate Statistics (3rd ed.). New York: Harper Collins.

[23]. Tô Linh, (2021, ngày 29 tháng 1). [Báo cáo] Nhu cầu sủ dụng dịch vu giao nhận đồ ăn của người Việt mùa dịch Covid-19. Truy xuất từ: https://marketingai.admicro.vn/bao-cao-nhu-cau-su-dung-dich-vu-giao-nhan-doan-cua-nguoi-viet-mua-dich-covid-19/

[24]. Trần Kim Anh, (2020, ngày 15 tháng 7). Phí sinh hoạt ở Việt Nam tăng 5 bậc, TP.HCM thành nơi đắt đỏ nhất nước. Truy xuất từ: https://thanhnien.vn/doi-song/phi-sinh-hoat-o-viet-nam-tang-5-bac-tphcm-thanh-noi-datdo-nhat-nuoc-1251778.html

[25]. Javadi, M. M. H., Dolatabadi, H. R., Nourbakhsh, M., Poursaeedi, A. \& Asadollahi, A. R. (2012). An Analysis of Factors Affecting on Online Shopping Behavior of Consumers, International Journal of Marketing Studies, $4(5), 81$.

[26]. Venkatesh, V., \& Davis, F. D. (2000). A theoretical extension of the technology acceptance model: Four longitudinal field studies. Management science, 46(2), 186-204.

[27]. Webster, J., Trevino, L. K., \& Ryan, L. (1993). The dimensionality and correlates of flow in human-computer interactions. Computers in human behavior, 9(4), 411-426.

[28]. Xu hướng sử dụng Úng dụng giao hàng thức ăn tại Việt Nam 2020. Truy xuất từ: https://qandme.net/vi/baibaocao/xu-huong-su-dung-ung-dung-giao-hang-thuc-an-tai-vietnam-2020.html

[29]. Yen, R., \& Gwinner, K. P. (2003). Internet Retail Customer Loyalty: The Mediating Role of Relational Benefits. International Journal of Service Industry Management, 14, 483-500.

Ngày nhận bài: 17/05/2021

Ngày chấp nhận đăng: 23/07/2021 